\title{
NEFRECTOMIAA DEL DONANTE VIVO: TÉCNICA DE LA UNIVERSIDAD DE MIAMI Y RESULTADOS ACTUALES
}

\author{
Samir P. Shirodkar4, Junichiro Sageshima',2, Vincent G. Bird4', Jose M. Martinez 1,2,3, Linda \\ Chen ${ }^{1,2}$, George W. Burke ${ }^{1,2}$ y Gaetano Ciancio 1,2,4,5.
}

Departamento de Cirugía', División de Trasplantes², y División de Cirugía Laparoendoscópica y Bariátrica ${ }^{3}$, Departamento de Urología4, Facultad de Medicina Universidad de Miami. Miami. Florida.

${ }^{5}$ Department of Surgery. University of Miami Miller School of Medicine. Miami. Florida.

Resumen.- OBJETIVO: La nefrectomía del donante vivo ha aumentado significativamente el fondo de donantes para trasplante renal, permitiendo un incremento marcado de los trasplantes. Las mejorías de la medicación contra el rechazo y el refinamiento de los criterios de selección del donante han permitido unas tasas de supervivencia del injerto extremadamente favorables. Más recientemente, la nefrectomía laparoscópica del donante vivo (NLDV) ha reducido significativamente la morbilidad en la población de donantes. La Universidad de Miami/Centro de Trasplantes del Hospital Jackson Memorial ha realizado un gran número de nefrectomías del donante vivo, con un aumento de la utilización de la nefrectomía laparoscópica del donante vivo y en el

\section{CORRESPONDENCIA}

Gaetano Ciancio

Departamneto de Cirugía

División de transplante

University of Miami Miller School of Medicine

Post Office Box 012440

Miami, FL 33101 (USA)

gciancio@med.miami.edu

Aceptado para publicar: 10 de noviembre 2009 presente artículo comunicamos nuestra experiencia acumulada.

MÉTODOS: Realizamos una revisión retrospectiva de todas las nefrectomías del donante vivo realiza las durante los últimos diez años, incluyendo las nefrectomías laparoscópicas. Se recogieron las complicaciones quirúrgicas, tanto menores como graves. También se registraron las conversiones de laparoscopia cirugía abierta. Se revisó el seguimiento, incluyendo las creatininas al año del trasplante en los grupos de nefrectomía abierta del donante y laparoscópica.

RESULTADOS: Se han realizado 413 nefrectomías del donante vivo durante diez años. De éstas, 257 fueron laparoscópicas y 156 abiertas. En dos casos, la nefrectomía laparoscópica fue convertida a abierta. Tres donantes necesitaron de intervención después de la nefrectomía. No hubo ninguna muerte perioperatoria ni trombosis venosa profundas. Las complicaciones menores, incluyendo hernia, fiebre y diarrea por C.difficile fueron muy raras, siendo la más frecuente el dolor testicular en ocho casos.

CONCLUSIONES: Nuestra amplia experiencia en nefrectomía del donante vivo, con 413 casos a lo largo de diez años, ha sido muy favorable. El riesgo de complicaciones graves fue extremadamente bajo, con seis casos comunicados en esta serie. Las complicaciones menores también fueron raras. La nefrectomía del donante vivo es un método seguro y factible de aumentar el número de donantes para trasplante renal con mínima morbilidad.

Palabras clave: Nefrectomía del donante. Donante vivo. Trasplante renal. Nefrectomía. Manoasistida. 
Summary.- OBJECTIVES: Living-donor nephrectomy has significantly expanded the pool of renal transplant donors, allowing for a marked increase in transplantation. Improvements in antirejection medications and refinement of donor selection criteria have allowed for extremely favorable rates of graft survival. More recently, laparoscopic donor nephrectomy (LDN) has significantly reduced the morbidity of renal transplantation in the donor population. The University of Miami/Jackson Memorial Hospital Transplant Center performs a large number of living-donor nephrectomies, with increasing use of LDN and here we report our cumulative experience.

METHODS: A retrospective review was performed of all live donor nephrectomies performed over the last 10 years, including LDN. Surgical complications, both minor and major, were ascertained. Conversion from LDN to open was similarly noted. Follow up, including creatinine one year post-transplant was recorded in open donor nephrectomy (ODN) and LDN groups.

RESULTS: Over 10 years, 413 live donor nephrectomies were performed. Of these, 257 were LDN, and 156 were ODN. In two cases, LDN was converted to ODN. Three patients needed reoperation after donor nephrectomy. There were no perioperative mortalities or deep venous thrombosis. Minor complications, including hernia, fever, and C. difficile diarrhea were very rare, the most common being testicular pain in eight patients.

CONCLUSION: Our extensive experience with living donor nephrectomy, with 413 cases spanning ten years, has been very favorable. The risk of major complications was extremely low, with six reported in the series. Minor complications were similarly rare. Living donor nephrectomy is a safe and feasible method of increasing the number of renal transplantation donors with minimal morbidity.

Keywords: Donor nephrectomy. Living donor. Renal transplantation. Nephrectomy. Hand-assisted.

\section{INTRODUCCIÓN}

La primera nefrectomía del donante vivo llevará a cabo con éxito fue en 1954 entre gemelos (1). En aquel tiempo, ni la histocompatibilidad ni la inmunosupresión habían sido descritas y los intentos de trasplante anteriores terminaron con la pérdida rápida del injerto.

El trasplante ofrece ventajas significativas a los pacientes con enfermedad renal terminal en comparación con la diálisis crónica, incluyendo disminución del riesgo de muerte, disminución del coste eco- nómico y un marcado aumento de la calidad de vida $(2,3)$. Desafortunadamente, el número de donantes disponibles es muy inferior al número de pacientes esperando trasplante. Durante muchos años la inmensa mayoría de los trasplantes se realizaron con órganos de cadáver. Sin embargo, por primera vez en 2001, el número de donantes vivos sobrepasó al de donantes cadáver (4).

Como resultado, se ha prestado mayor atención a las nefrectomías del donante vivo, incluyendo la técnica (5), los cuidados perioperatorios y el seguimiento a largo plazo.

El Instituto de Trasplantes de Miami (Miami Transplant Institute-MTI) en la Universidad de Miami/ Hospital Jackson Memorial es uno de los centros de trasplante renal con mayor volumen de los Estados Unidos. Aproximadamente el $28 \%$ de los trasplantes renales de Florida se realizan en el MTI (6). En el presente artículo comunicamos nuestra experiencia con el trasplante renal con donante vivo en los últimos diez años.

\section{MATERIAL Y MÉTODOS}

\section{Técnicas}

Se identificaron los pacientes sometidos a cualquier forma de nefrectomía del donante vivo durante los últimos diez años, incluyendo nefrectomía laparoscópica mano asistida del donante vivo (NLDV) y nefrectomía abierta(NADV). Se llevó a cabo una revisión retrospectiva de las historias clínicas para evaluar los factores pre-, intra- y postoperatorios. Se registraron los valores de creatinina perioperatoria y postoperatoria. Las complicaciones perioperatorias fueron registradas en base a la revisión de las historias. Se midió la creatinina postoperatoria en la cohorte de donantes durante al menos un año. Estos valores fueron registrados y se realizó una comparación entre los pacientes sometidos a nefrectomía laparoscópica y nefrectomía abierta.

La nefrectomía abierta se realiza conforme a la técnica previamente descrita (7). Brevemente, con el paciente en decúbito lateral y la mesa de operaciones flexionada al máximo, se realiza una incisión desde la punta de la $11^{\underline{a}} \circ 12^{\underline{a}}$ costilla en dirección anterior hacia el ombligo llegando hasta el borde lateral del músculo recto abdominal. La longitud de la incisión cutánea ha sido recientemente minimizada hasta $10-15 \mathrm{~cm}$, abordaje conocido como nefrectomía del donante vivo mini-incisional, y no es necesaria la escisión de la costilla en la mayoría de los casos. Después de la disección de los planos musculares se colocan separadores mecánicos y se recha- 
za el peritoneo medialmente para acceder al espacio retroperitoneal. Posteriormente, se abre la fascia de Gerota y se diseca el riñón de la grasa perirenal. Una disección cuidadosa del hilio renal asegura unos vasos renales intactos. Para prevenir el espasmo arterial y preservar un buen flujo sanguíneo al riñón se utiliza frecuentemente papaverina tópica y manitol sistémico antes de la extracción. El uréter se diseca y divide a nivel de las arterias ilíacas. Después de la heparinización, se aplican clamps vasculares a la arteria $(s)$ y vena(s) renales y se dividen secuencialmente. El riñón se extrae del campo y es perfundido utilizando solución de Ringer suplementada helada. Se revierte la heparina mediante protamina y se suturan los vasos con puntos continuos de polipropileno. La incisión se cierra por planos.

La nefrectomía del donante laparoscópica mano asistida se inicia por la inducción de anestesia general. El paciente es colocado en posición de decúbito lateral derecho a $30^{\circ}$. El paciente es colocado de tal forma que la flexión de la mesa y la elevación del soporte lumbar consigan la mejor exposición posible del flanco izquierdo. Se utiliza el riñón izquierdo en todos los casos de laparoscopia del donante debido a la mayor longitud de la vena renal. Realizamos una incisión periumbilical y a continuación se inicia neumoperitoneo. Preferimos utilizar $15 \mathrm{mmHg}$. A continuación colocamos los trocares. Inicialmente colocamos el trocar de $10 \mathrm{~mm}$ de la cámara y a continuación dos o tres adicionales bajo visión directa. $\mathrm{Se}$ coloca uno en la línea media axilar subcostal. Otro en la mitad de la línea que une el trocar umbilical con la cresta iliaca. Ocasionalmente, colocamos un trocar de $5 \mathrm{~mm}$ para el asistente, craneal al tercer trocar para obtener retracción adicional y ayuda en la exposición.

Se abre el peritoneo y se continúa la incisión hacia abajo siguiendo la línea de Toldt hasta reflejar medialmente el colon descendente. De forma similar se extiende la incisión peritoneal en sentido cefálico hacia en el pilar del diafragma.

Una modificación importante que utilizamos es la movilización en bloque del bazo y el páncreas con el colon durante la nefrectomía laparoscópica. La movilización del bazo comienza con la disección de las fijaciones peritoneales posteriores. Se moviliza el bazo y se utiliza como asa para levantar la cola del páncreas. Se debe tener un cuidado extremo para no ejercer una tracción indebida sobre el bazo. Debe retraerse en dirección medial, lo cual expone tanto los ligamentos lienodiafragmáticos como lienorenales. Estos deben dividirse con electrocauterio antes de continuar la disección. Debe separarse pronto el bazo de la fascia de Gerota y del diafragma con di- sección roma para prevenir un neumotórax. El cirujano refleja el bazo y el colon izquierdo hacia la línea media y al mismo tiempo incide el plano fibroadiposo avascular alrededor de un $\mathrm{cm}$ desde el aspecto posterior de la cápsula pancreática. Esto ayuda a separar el páncreas del riñón. Los siguientes puntos son importantes: visualizar y preservar la arteria y vena esplénicas utilizando el bazo como asa (como se ha descrito anteriormente). Evitar una tracción innecesaria del bazo que puede llegar producir una rotura de su cápsula de sangrado. Evitar la entrada en el cuerpo del páncreas durante la disección. Evitar la lesión de la serosa gástrica al movilizar el aspecto posterior del estómago. Identificar y preservar la vena mesentérica inferior en el borde inferior del páncreas. Mantenerse fiel al principio de movilización en bloque más que disección individual de los órganos. Con tal fin, el bazo debe permanecer fijo a la curvatura mayor del estómago por la gastroepiploica izquierda y los vasos gástricos cortos. Al completar la movilización, el riñón y la glándula suprarrenal quedan expuestos para continuar la disección. La glándula suprarrenal se separa del riñón después de ligar los vasos adrenales. Para prevenir la devascularización ureteral, se toma el uréter en bloque con la vena gonadal y la grasa y tejidos periureterales. Posteriormente, el resto del procedimiento se continúa en la forma tradicional mano asistida (5), incluyendo la división de los vasos renales después de la administración de heparina y su reversión con protamina.

\section{RESULTADOS}

En un periodo de diez años, desde 1998 al 2008, se han realizado 413 nefrectomías del donante vivo. De ellas 156 fueron nefrectomías abiertas y 257 laparoscópicas. La Figura 1 muestra la tenden-

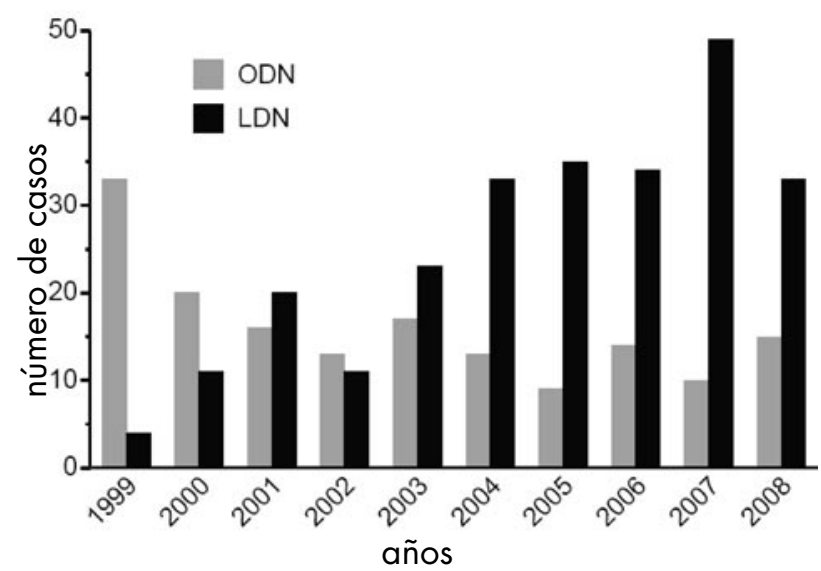

FIGURA 1. Número de casos por año de nefrectomía abierta del donante vivo (ODN) comparados con la nefrectomía laparoscópica del donante vivo (LDN). 
cia operatoria comparando NADV y NLDV. No se han registrado muertes perioperatorias. En el periodo de diez años se han registrado seis complicaciones graves: hubo dos conversiones de NLDV a NADV. La tasa de conversión fue del $0,8 \%$. Tres pacientes requirieron reintervención, y un paciente transfusión por anemia postoperatoria pero no sin evidencia de sangrado activo. $1,4 \%$.

La tasa de complicaciones graves fue del

En los casos de conversión de laparoscopia a abierta, un caso se debió a la preferencia del cirujano por una anatomía adversa en relación con múltiples arterias. La segunda fue debido a una glándula suprarrenal adherente y mala función del instrumental (grapado vascular incompleto). De los tres pacientes que requirieron reintervención, la obstrucción de intestino delgado parcial o completa fue la indicación en dos, manejada en ambos casos con laparoscopia. El tercer paciente fue intervenido por sangrado postoperatorio- el clip Hemolock (WECK) se había salido del muñón arterial. Todos los reoperados habían sido operados originalmente por laparoscopia. La tasa de complicaciones graves de la nefrectomía abierta del donante fue del $0 \%$ y la de la nefrectomía laparoscópica del donante vivo del $1,4 \%$.

Se registraron complicaciones menores en 28 pacientes, con una tasa global del 6,7\%. Las siguientes aparecieron cada una en un paciente: anemia que no requirió transfusión (NLDV), neumotórax (NADV), ascitis quilosa (NLDV), pancreatitis(NLDV), y rabdomiolisis (NLDV). Aparecieron en dos pacientes cada una de las siguientes: celulitis/infección de la herida (una NLDV, una NADV), diarrea por C. difficile (una NLDV, una NADV), hernia incisional (una NLDV, una NADV), y derrame pleural (ambas NADV). Tres pacientes tuvieron fiebre en el posoperatorio (dos NADV, uno NLDV). Cuatro pacientes reingresaron por dolor e íleo (todos NLDV). Finalmente, ocho pacientes, todos de la cohorte laparoscópica padecieron dolor testicular postoperatorio. Cuando las separamos por técnica, la tasa complicaciones menores de la nefrectomía abierta fue de $5,1 \%$ y de la laparoscópica el 7,8\%. La Tabla I representa todas complicaciones de la serie de nefrectomía en donante de nuestra institución.

Dado que se realizaron un gran número nefrectomías laparoscópicas del donante vivo además de las nefrectomías abiertas, nuestro análisis también incluye una comparación de las dos modalidades. La creatinina preoperatoria en los grupos de NLDV y NADV fue de 0,86 y $0,87 \mathrm{ng} / \mathrm{dL}$ respectivamente $(p=0,82)$. A las tres semanas de la operación, los valores de creatinina fueron de 1,26 ng/dL (NLDV) y $1,21 \mathrm{ng} / \mathrm{dl}$ (NADV) $(p=0,23)$. En la última visita de seguimiento a las 52 semanas postoperatorias, seguía sin haber diferencias significativas en los valores de creatinina entre los grupos. La Figura 2 representa estos valores.

\section{DISCUSIÓN}

La nefrectomía del donante vivo tiene un número significativo de ventajas sobre el trasplante de cadáver (8). Como muestra, los costes de la pérdida del injerto incluyendo reinicio de la diálisis, reevaluación para trasplante, y retrasplante se reducen de una forma marcada, dando como resultado un gasto significativamente menor en los pacientes sometidos a trasplante con donante vivo en comparación con el donante cadáver (9).

La primera nefrectomía del donante vivo fue realizada en 1954 entre gemelos idénticos. Anteriormente, los trasplantes experimentales habían fallado uniformemente debido a los obstáculos biológicos incluyendo, pero no limitada a, la incompatibilidad $A B O, H L A$ y de pruebas cruzadas (10). Avances posteriores en el campo mejoraron mucho la supervivencia de los injertos en trasplante renal no relacionado, particularmente entre esposos, demostrando investigaciones recientes sobre la supervivencia a largo plazo de los injertos resultados particularmente buenos (11).

Dada la mejoría de la supervivencia del injerto y el éxito a largo plazo en pacientes con enferme-

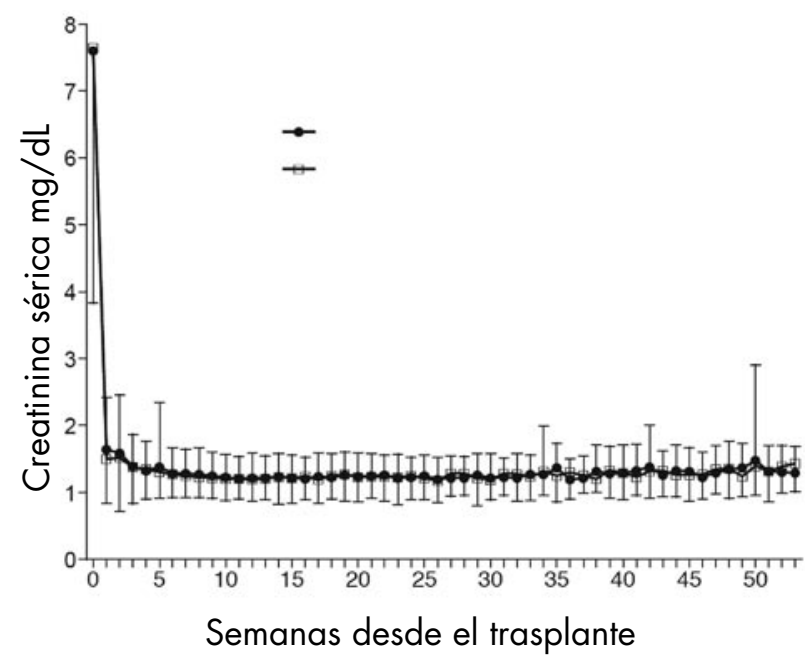

FIGURA 2. Valores de creatinina en trasplantes de NLDV comparados con NADV. 
dad renal terminal, se ha puesto mucha atención en el donante de riñón. Durante más de 35 años desde la nefrectomía inicial realizada por Murray en 1954, la donación renal de vivo se ha realizado utilizando un abordaje quirúrgico abierto. Hasta mediados de los 90 hubo modificaciones mínimas de la técnica quirúrgica en el abordaje abierto de la nefrectomía del donante vivo. Varios autores han comunicado su experiencia con incisiones de mini laparotomía en comparación con lumbotomía en este grupo de pacientes (12-14). La mayoría de las comparaciones muestran una diferencia mínima entre estos abordajes, con algunos grupos que comunican mejoría de las puntuaciones de dolor y estancias más cortas en los pacientes sometidos a nefrectomía del donante mini incisional en comparación con la nefrectomía del donante por lumbotomía tradicional (14). Nuestro abordaje abierto ha sido la lumbotomía ortodoxa

\section{TABLA I. COMPLICACIONES GRAVES Y LEVES DE LA NEFRECTOMÍA DEL DONANTE VIVO.}

\begin{tabular}{|c|c|c|}
\hline Tipo & Número abiertas & Número laparoscópicas \\
\hline 1. Conversión de laparoscopia cirugía abierta & 0 & 2 \\
\hline 2. Reoperación & 0 & 3 \\
\hline 3. Anemia con transfusion & 0 & 1 \\
\hline 4. Muerte & 0 & 0 \\
\hline 5. Trombosis venosa profunda & 0 & 0 \\
\hline Número Total de Complicaciones Graves & 0 & 6 \\
\hline \multicolumn{3}{|c|}{ Leves } \\
\hline 1. Anemia que no requiere transfusión & 0 & 1 \\
\hline 2. Neumotórax & 1 & 0 \\
\hline 3. Celulitis/infección de la herida & 1 & 1 \\
\hline 4. Diarrea por C. difficile & 1 & 1 \\
\hline 5. Fiebre & 2 & 1 \\
\hline 6. Dolor Testicular & 0 & 8 \\
\hline 7. Hernia & 1 & 1 \\
\hline 8. Derrame pleural & 2 & 0 \\
\hline 9. Reingreso por dolor, ileo prolongado & 0 & 4 \\
\hline 10. Ascitis quilosa & 0 & 1 \\
\hline 11. Pancreatitis & 0 & 1 \\
\hline 12. Rabdomiolisis & 0 & 1 \\
\hline Número total de complicaciones leves & 8 & 20 \\
\hline
\end{tabular}


con transición hacia la mini incisión. Este último abordaje ha tenido éxito con mínimas complicaciones y morbilidad en los pacientes. Ha habido dos hernias incisionales en relación con este abordaje. Ninguna de ellas requirió de operación o tratamiento invasivo. Este dato es comparable con otros centros que comunican entre el $3,6 \%$ y el $7 \%$ de incidencia de hernia en la nefrectomía abierta del donante $(15,16)$. Nuestra experiencia con dolor postoperatorio, el inicio de la dieta, las estancias hospitalarias y la satisfacción del paciente han sido excelentes (datos no presentados) y similares a los presentados por otros centros $(17,18)$.

La primera nefrectomía laparoscópica realizada en 1991 por Clayman y cols. (19) inició un cambio significativo de la técnica de nefrectomía del donante vivo. Por primera vez en el humano, la nefrectomía laparoscópica había demostrado ser segura, bien tolerada, con mínima morbilidad adicional relacionada con el procedimiento. Posteriormente, la nefrectomía laparoscópica fue aplicada por Gill y cols en un modelo porcino de nefrectomía del donante (20). El éxito de este modelo proporcionó el ímpetu para la transición a estas técnicas en la nefrectomía del donante en el humano. La comunicación inicial de nefrectomía del donante vivo humano, por Ratner y cols. en 1995 fue un hito en el campo de la nefrectomía del donante (21). Las modificaciones posteriores y las comparaciones iniciales confirmaron que este método era seguro, eficaz y no tenía ningún efecto perjudicial sobre la supervivencia del injerto (22-24).

Desde estas comunicaciones iniciales, se ha acumulado un vasto cuerpo de literatura que proporciona una evidencia fuerte sobre la viabilidad de la nefrectomía laparoscópica del donante vivo. Gran parte de la literatura está enfocada en tres aspectos de la NLDV en comparación con la NADV: las variables intraoperatorias, las diferencias postoperatorias y los resultados a largo plazo del injerto.

La mayoría de los autores comunican una diferencia estadísticamente significativa de la duración de la operación comparando NADV con NLDV. La inmensa mayoría de las fuentes comunican que la NADV se realizará de una forma generalmente más rápida que la NLDV $(18,25,26)$. Varios autores han comunicado un aumento de las complicaciones en las cohortes de NLDV en comparación con los pacientes de NADV (24-26). Sin embargo, estas publicaciones deben ser evaluadas en el contexto de la curva de aprendizaje de la técnica laparoscópica en comparación con la abierta. De hecho, Jacobs y cols. comunicaron que a lo largo de tres años de su experiencia con NLDV tanto el tiempo operatorio como la incidencia de complicaciones disminuyeron significativamente. Las pérdidas sanguíneas durante estos casos también han sido comparadas mostrando mínimas diferencias. La mayoría de las fuentes están de acuerdo con que el abordaje laparoscópico tiene menor sangrado (18), sin embargo, el potencial de pérdida masiva de sangre es mayor en estos casos dado el (raro) riesgo de mal funcionamiento del equipo incluyendo fallos de disparo de las endocortadoras y fallos de los clips.

En el postoperatorio, los pacientes sometidos a NLDV parecen tener varias ventajas frente a los de la NADV. Casi todas estas fuentes están de acuerdo en que los requerimientos medios de analgésicos postoperatorios se reducen de una forma macada en los pacientes después de la cirugía laparoscópica en comparación con el abordaje abierto $(23,24$, $27,28)$. El inicio de la tolerancia oral, la estancia hospitalaria y el retorno a la actividad normal mejoran también significativamente en los pacientes de NLDV en comparación con los que fueron sometidos a NADV $(23,28)$.

Finalmente, y de máxima importancia, parece que no existe diferencia significativa en la función del injerto en receptores de donantes sometidos a NLDV o NADV $(23,30,31)$. Todos estos autores comunican que no hubo diferencias entre los grupos en los niveles de creatinina en el postoperatorio precoz y a largo plazo. Algunos autores han sugerido que dada una ligera reducción de la isquemia caliente y del tiempo quirúrgico la función precoz del injerto era mejor pero tales publicaciones no han demostrado resultados estadísticamente significativos.

Nuestra experiencia ha sido similar en todos los aspectos a la de los trabajos publicados. No hemos tenido muertes intraoperatorias o postoperatorias, ni trombosis venosas profundas. La tasa de conversión a cirugía abierta menor del 1\% compara favorablemente con otras publicaciones entre el $1 \%$ y el $6,3 \%(25,26,29)$. No hemos comunicado los tiempos operatorios, sin embargo nuestra experiencia refleja la de otros grupos en relación con las técnicas abiertas y laparoscópicas. En nuestra experiencia tres pacientes requirieron reintervencion. Dos presentaron obstrucción de intestino delgado parcial o total se necesitó exploración quirúrgica para su resolución. Un paciente fue operado por sangrado intrabdominal. En el momento de la exploración, el clip Hemolock (Weck) laparoscópico se salió del muñón de la arteria renal. Como resultado de esta complicación, hemos modificado nuestra técnica incluyendo aparatos de grapado penetrante siguiendo las recomendaciones de numerosas organizaciones y no hemos tenido más complicaciones. 
La tasa de complicaciones menores del $6,7 \%$ también era similar a la comunicada por otros centros. Dos pacientes presentaron celulitis e infección de la herida requiriendo antibióticos y evaluación de las heridas. Se constató que el plano de la fascia estaba intacto y no hizo falta tratamiento quirúrgico en estos pacientes. Cuatro pacientes necesitaron reingreso-por dolor e ileo prolongado. Ninguno de estos pacientes necesitó tratamiento invasivo. Interesantemente, la complicación menor más común registrada fue dolor testicular en ocho pacientes. Ésta parece ser una complicación vista frecuentemente, comunicada previamente por otros centros 132,33 . La etiología es desconocida, sin embargo las hipótesis incluyen lesión de los nervios sensitivos del testículo durante la disección ureteral o posible sección del cordón espermático. La ligadura de los vasos gonadales también puede contribuir a este hallazgo en donantes varones. En todos los casos comunicados, el dolor mejoró espontáneamente sin ninguna intervención.

Nuestra transición desde la incisión de lumbotomía ortodoxa a mini incisión también es destacable. La diferencia de la longitud de la incisión con la del sitio del dispositivo Hand port laparoscópico es mínima, con mejoría del dolor y del resultado cosmético para el paciente nuestra experiencia. No hemos registrado complicaciones durante esta transición. La proporción de NLDV y NADV ha ido cambiando sin cesar con el tiempo (Tabla I). Al inicio del programa de donante vivo, casi todos los casos se hacían utilizando la técnica abierta. Desde entonces ha habido un aumento continuo del número de procedimientos laparoscópicos y más recientemente casi el $75 \%$ de todas las cirugías del donante se hicieron con esa técnica. Todas las nefrectomías del donante fueron izquierdas. No anticipamos una reversión de esta tendencia en un futuro próximo. La función del injerto medida por la creatinina postoperatoria no ha mostrado diferencias estadísticas entre los grupos de NLDV y la NADV en ningún momento del postoperatorio.

\section{CONCLUSIONES}

La nefrectomía del donante vivo ha revolucionado el trasplante renal y, por extensión, la enfermedad renal terminal. El tratamiento de la enfermedad renal terminal por medio del trasplante tiene múltiples ventajas para el receptor, y el número de pacientes que reciben un trasplante renal se ha incrementado significativamente. Las técnicas de nefrectomía del donante vivo se han refinado mucho desde la primera nefrectomía del donante en los 50. Actualmente se realizan menos nefrectomías abiertas del donante vivo, siendo reemplazadas por un número mayor de nefrectomías laparoscópicas. Las ventajas para el donante son numerosas, incluyendo mejor cosmética, menor estancia hospitalaria y menor dolor. Nuestra experiencia con la nefrectomía del donante vivo ha reflejado estos cambios en la técnica. Actualmente, casi las tres cuartas partes de todas las nefrectomías del donante se realizan por laparoscopia. La tasa complicaciones es mínima y los resultados a largo plazo del injerto son excelentes.

\section{BIBLIOGRAFIA y LECTURAS RECOMENDADAS ( ${ }^{*}$ lectura de interés $y^{* *}$ lectura fundamental)}

*1. Murray JE, Merrill JP, Harrison JH. Renal homotransplantation in identical twins. Surg Forum 1955; 6:432.

**2. Wolfe RA, Ashby VB, Milford EL, et al. Comparison of mortality in all patients on dialysis awaiting transplantation, and recipients of a first cadaveric transplant. N Engl J Med. 1999; 341:1725-30.

3. Laupasis A, Keown P, Pus N, et al. A study of the quality of life and cost-utility of renal transplantation. Kidney Int. 1996; 50:235-42.

4. Davis CL. Evaluation of the living kidney donor: current perspectives. Am J Kidney Dis. 2004; 43(3):508-30.

5. Skrekas G, Papalois VE, Mitsis M, Hakim NS. Laparoscopic live donor nephrectomy: a step forward in kidney transplantation? JSLS. 2003; 7(3):197-206.

6. www. Miamitransplantinstitute . com

7. Barry JM, Jordan ML, and Conlin MJ, Renal Transplantation. In: A.J. Wein, L.R. Kavoussi, A.C. Novick, A.W. Partin and C.A. Peters, Editors, Campbell-Walsh Urology (9th ed.), Saunders Elsevier, Philadelphia, PA (2007), pp. 1295-1324.

8. Cecka JM. The UNOS scientific renal transplant registry-ten years of kidney transplants. In: Cecka JM, Terasaki PI, eds. Clinical Transplants 1997. UCLA Tissue Typing Laboratory, Los Angeles; 1998; 1

*9. Eggers P. Comparison of treatment costs between dialysis and transplantation. Semin Nephrol 1992; 12: 284-9.

10. Davis CL, Delmonico FL. Living-donor kidney transplantation: a review of the current practices for the live donor. J Am Soc Nephrol. 2005; 16(7):2098-110.

11. Terasaki PI, Cecka JM, Gjertson DW, Cho YW. Spousal and other living renal donor transplants. Clin Transpl. 1997:269-84.

12. Neipp M, Jackobs $\mathrm{S}$, Becker T, zu Vilsendorf AM, Winny M, Lueck R, Klempnauer J, Nashan B. Living donor nephrectomy: flank incision versus 
anterior vertical mini-incision. Transplantation. 2004; 78(9):1356-61.

13. Kok NF, Alwayn IP, Lind MY, Tran KT, Weimar W, IJzermans JN. Donor nephrectomy: mini-incision muscle-splitting open approach versus laparoscopy. Transplantation. 2006; 81(6):881-7.

14. Schnitzbauer AA, Loss M, Hornung M, Glockzin G, Mantouvalou L, Krüger B, Krämer BK, Schlitt HJ, Obed A. Mini-incision for strictly retroperitoneal nephrectomy in living kidney donation vs flank incision. Nephrol Dial Transplant. 2006; 21(10):2948-52.

15. Bayazit Y, Aridogan IA, Tansug Z, Unsal I, Erken U. Morbidity of flank incision in 100 renal donors. Int Urol Nephrol. 2001; 32(4):709-11.

16. Dunn JF, Nylander WA Jr, Richie RE, Johnson HK, MacDonell RC Jr, Sawyers JL. Living related kidney donors. A 14-year experience. Ann Surg. 1986; 203(6):637-43.

17. Flowers JL, Jacobs SC, Cho E, et al. Comparison of open and laparoscopic live donor nephrectomy. Ann Surg. 1997; 226:483-90.

**18. Wolf JS Jr, Marcovich R, Merion RM, Konnak JW. Prospective, case matched comparison of hand assisted laparoscopic and open surgical live donor nephrectomy. J Urol. 2000; 163:1650-3.

*19. Clayman RV, Kavoussi LR, Soper NL, et al. Laparoscopic nephrectomy. New Engl J Med. 1991; 324:1370-1.

20. Gill IS, Carbone JM, Clayman RV, et al. Laparoscopic livedonor nephrectomy. J Endourol. 1994; 8:143-8.

21. Ratner LE, Ciseck LJ, Moore LG, et al. Laparoscopic live donor nephrectomy. Transplantation. 1995; 60:1047-9.

22. Fabrizio MD, Ratner LE, Montgomery RA, Kavoussi LR. Laparoscopic live donor nephrectomy. Urol Clin North Am. 1999; 26:247-56.

23. Flowers JL, Jacobs SC, Cho E, et al. Comparison of open and laparoscopic live donor nephrectomy. Ann Surg. 1997; 226:483-90.
**24. Ratner LE, Kavoussi LR, Schulam PG, Bender JS, Magnuson TH, Montgomery R. Comparison of laparoscopic live donor nephrectomy versus the standard open approach. Transplant Proc. 1997; 29:138-9.

25. Oyen O, Andersen M, Mathisen L et al. Laparoscopic versus open living-donor nephrectomy: experiences from a prospective, randomized, single-center study focusing on donor safety. Transplantation 2005; 79: 1236-40

**26. Simforoosh N, Basiri A, Tabibi A, Shakhssalim N, Hosseini Moghaddam SM. Comparison of laparoscopic and open donor nephrectomy: a randomized controlled trial. BJU Int 2005; 95: 851-5.

27. Jacobs SC, Cho E, Dunkin BJ, et al. Laparoscopic live donor nephrectomy: the University of Maryland 3-year experience. J Urol. 2000;164:14949.

*28. Leventhal JR, Deeik RK, Joehl RJ, et al. Laparoscopic live donor nephrectomy-is it safe? Transplant. 2000;70(4):602-6.

29. Rawlins MC, Hefty TL, Brown SL, Biehl TR. Learning laparoscopic donor nephrectomy safely: a report of 100 cases. Arch Surg 2002; 137: 5315.

30. Odland MD, Ney AL, Jacobs DM, et al. Initial experience with laparoscopic live donor nephrectomy. Surgery. 1999; 126:603-6.

31. London E, Rudich S, McVicar J, Wolfe B, Perez R. Equivalent renal allograft function with laparoscopic versus open live donor nephrectomies. Transplant Proc. 1999; 31:258-60.

32. Kim FJ, Pinto P, Su LM, Jarrett TW, Rattner LE, Montgomery R, Kavoussi LR. Ipsilateral orchialgia after laparoscopic donor nephrectomy. J Endourol. 2003; 17(6):405-9.

33. Gjertson CK, Sundaram CP. Testicular pain following laparoscopic renal surgery. J Urol. 2008; 180(5):2037-40. 